\title{
Linear correlation of vertical ionization energies and partial charges on acetaldehyde and methyl formate radicals in various solvents
}

\author{
Constantinos D. Zeinalipour-Yazdi ${ }^{a, b} *$ Kevin $\operatorname{Lam}^{a}$ \\ ${ }^{a}$ School of Science, University of Greenwich, Central Avenue, Chatham Maritime, \\ Kent, ME4 4TB, UK \\ ${ }^{b}$ Department of Chemistry, University College London, 20 Gordon Street, London, \\ WCIH OAJ, UK
}

Correspondence to: c.zeinalipouryazdi@greenwich.ac.uk

\begin{abstract}
We have studied the vertical ionisation energy (IE) and the partial charge on the carbonyl carbon atom of acetaldehyde and methyl formate radicals, in various solvents (i.e. cyclohexane, benzene, methanol, ethanol, DMSO, water, acetonitrile, THF, chloroform). We find a linear correlation between the IE and the partial charge on the carbonyle carbon of these radicals. This suggests that there is a small solvent effect on the rate of radical to carbocation formation for radicals such as acetaldehyde and methyl formate radicals, which can be explained on a simplified point charge model following Coulomb's law.
\end{abstract}

\section{Introduction}

Recent progress in synthetic photoredox and electrochemistry has shown a vivid interest in the chemistry of ketyl and oxocarbonyl radicals and their use for the preparation of pharmaceutically relevant compounds. The photochemical or anodic oxidation of hemioxalate and alphaketo-acids lead to the decarboxylative formation of an $\mathrm{sp}^{2}$ centered radical. ${ }^{1}$ Nevertheless, there is a striking difference between the photoredox and electrochemical methods. Indeed, when a photoredox electrontransfer mediator is used, an acyl or oxocarbonyl radical is produced by decarboxylation of the corresponding derivative. However, under electrochemical 
conditions, the same compound could lead to the formation of the carbocation rather than that of the radical (see Fig. 1).

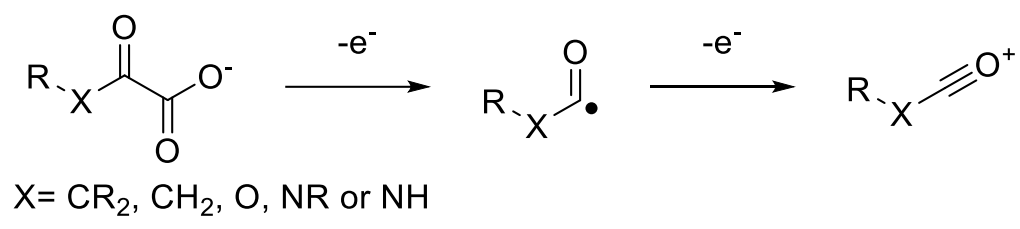

Fig. 1 Reaction showing the carbocation formation produced by decarboxylation of the corresponding derivative through an acyl or oxocarbonyl radical

Methyl formate has been widely used as an industrial solvent and blowing agent for foam isolation. ${ }^{2}$ The methyl formate radicals has been spectroscopically measured in interstellar medium molecular clouds. ${ }^{3}$ Industrially, small-scale methyl formate synthesis is carried out by a condensation reaction of methanol and formic acid given by the following reaction, $\mathrm{HCOOH}+\mathrm{CH}_{3} \mathrm{OH} \rightarrow \mathrm{HCO}_{2} \mathrm{CH}_{3}+\mathrm{H}_{2} \mathrm{O}$, however, industrial methyl formate synthesis is usually produced by the combination of methanol and carbon monoxide (see Fig. 2) in the presence sodium methoxide ${ }^{4}$ given by the following reaction,

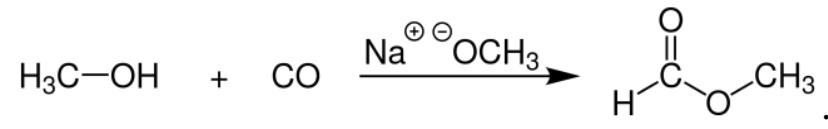

Fig. 2 Reaction showing industrial methyl formate synthesis

This is a strong base-catalyzed carbonylation of methanol with carbon monoxide to methyl formate commercially used by BASF with $96 \%$ selectivity. The carbon monoxide is derived from dry synthesis gas as the process is water sensitive. ${ }^{5}$ Methyl formate is a precursor for many commercial chemicals and therefore understanding its properties in solvents is very important. One example is the formation of radicals from methyl formate, which usually occurs via carbonyl $\mathrm{H}$ abstraction. Another important reaction is the formation of carbocations as the one shown in Fig. 3. A computational study of the reaction of the abstraction of carbonyl or methyl hydrogen from methyl formate to form the corresponding radical showed the carbonyl abstraction (shown in Fig. 3a) is favored by $84.6 \%$ at $298 \mathrm{~K}$ whereas at higher temperatures methyl hydrogen abstraction become equally possible. ${ }^{6}$ When the formation of the radical is mediated by $\mathrm{CH}_{3}$ the preference for the carbonyl radical becomes $98 \%$ clearly indicating that the Lewis structure for the radical is given in Fig. 
3. ${ }^{6}$ In an FTIR study methyl formate reaction with $\mathrm{Cl}$ resulted in $45 \% \mathrm{H}$ abstraction from the methyl group and 55\% abstraction from the $\mathrm{H}$ of the cabonyl group forming the corresponding radicals. ${ }^{7}$ The radicals formed were found to react with molecular oxygen forming the corresponding peroxo radicals, which in the case of the methyl formate radical would decompose to $\mathrm{CO}_{2}$ and methoxy radical. ${ }^{7} \mathrm{Ab}$ initio calculations coupled to flash photolysis UV spectroscopy experiment found that there are two absorption bands for the methyl formate radical in the region between $220 \mathrm{~nm}$ and $340 \mathrm{~nm} .{ }^{8}$ Using isotopically labelled methyl formate $\left(\mathrm{DCOOCH}_{3}\right)$ it was measured that at $182{ }^{\circ} \mathrm{C} 85 \%$ of the H/D abstraction occurs at the deuturated formyl group 9 in agreement with another study that measured the Arrhenius activation energies and pre-exponential factors for the two H-abstraction mechanisms. ${ }^{10}$

Acetaldehyde radicals have been detected in the interstellar medium ${ }^{11}$ and are generated atmospherically by the photodegradation of atmospheric secondary organic aerosol particles. ${ }^{12}$ They are considered one of the most abundant atmospheric carbonyls with concentrations at 100 ppt. $^{13}$ Acetaldehyde is a product of enthanol oxidation ${ }^{14}$ and its adverse human health effects ${ }^{15}$ have been linked to its formation of adducts with proteins, which increases due to smoking and alcohol consumption. Acetaldehyde is known to link to several proteins ${ }^{16}$ and to interfere with the physiological enzyme (e.g. carbonic anhydrase ${ }^{17}$ ) and cell function. ${ }^{18}$ Therefore chronic ethanol consumption can modify the hepatic proteins, which are the primary metabolite of ethanol, which has been linked to initiating alcoholic liver disease ${ }^{16}$ and carcinogenesis. ${ }^{19}$ Aldehyde-derived radicals are known to form in aqueous solutions and in cell in vivo. ${ }^{20}$ These radicals can through the loss of an electron from carbocations according to Fig. 3 and therefore understanding the properties of such radicals in various solvents is desirable in this study.

We have therefore studied how the ionisation energy changes as a function of the dielectric constant of various solvents (i.e. cyclohexane, benzene, methanol, ethanol, DMSO, water, acetonitrile, THF, chloroform) in order to understand whether there is an effect on their activity to form carbocations. We have used two computational methods (i.e. perturbation theory, density functional theory) with the use of implicit solvations models to report the IE and partial charges on the carbonyle carbon of two radicals (i.e. acetaldehyde and methyl formate). 
(a)

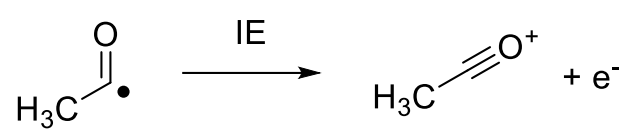

(b)

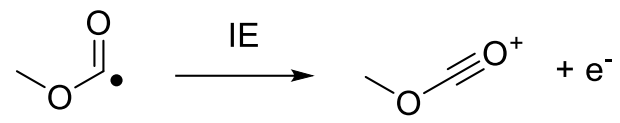

Fig. 3. (a) Acetaldehyde and (b) methyl formate radical and their potential mechanism of carbocation formation.

\section{Computational Methods}

All calculations were performed within the Gaussian 09 suit of programs using $\mathrm{UMP}^{21-23}$ and the UB3LYP 24,25 with an augmented correlation-consistent valence triple-zeta basis set, denoted as aug-cc-pVTZ(5d, 7f $)^{26-30}$, in implicit solvation using the polarizable continuum model (PCM) using the integral equation formalism variant (IEFPCM). ${ }^{31,32}$ Restricted wave functions are used for closed-shell and nonrestricted wave functions for open-shell systems. The possibility of spin-contamination was tested and the wavefunctions were found to not be significantly contaminated. For these doublet systems before annihilation spin contamination was found to be $\left\langle S^{2}\right\rangle=$ 0.7530 to 0.7536 not significantly different than the non-spin contaminated value of $\left\langle S^{2}\right\rangle=0.75$. Partial charges on the atoms were found using the atomic polar tensor (APT) method. This charge population analysis has the advantage of being invariant with respect to changes to the coordinate system. ${ }^{33}$

\section{Results and Disscusion}

\subsection{Electronic stucture of acetaldehyde and methyl formate radicals}

The radicals we have examined were acetaldehyde and methyl formate shown in Fig. 3 using the UB3LYP/aug-cc-pVTZ(5d, 7f) method in implicit solvation within the polarizable continuum model (PCM) using the integral equation formalism variant (IEFPCM). ${ }^{31,32}$ The singly occupied molecular orbital (SOMO) of the two radicals is shown in Fig. 4, which clearly shows that the shape of the SOMO is consistent with the common view that the singly occupied orbital protrudes at angle of roughly $120^{\circ}$ with respect to the carbonyl group having a mirror plane symmetry in the plane of the 
carbonyl group. The SOMO orbital for acetaldehyde appears to be somewhat bulkier than the one of the methyl formate radical for the same isodensity value (i.e. 0.02). This indicates that the lone electron is somewhat more stable in the SOMO of the acetaldehyde radical. This is latter also confirmed by the calculated values of the IE that shows the methyl formate radical has higher IE than the acetaldehyde radical.

(a)

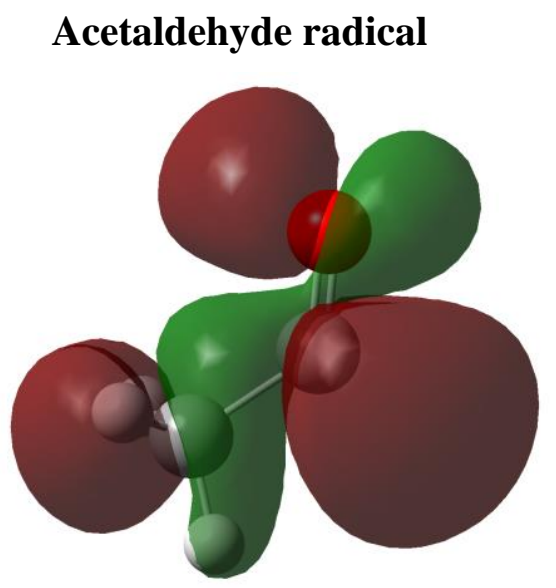

(b)

\section{Methyl formate radical}

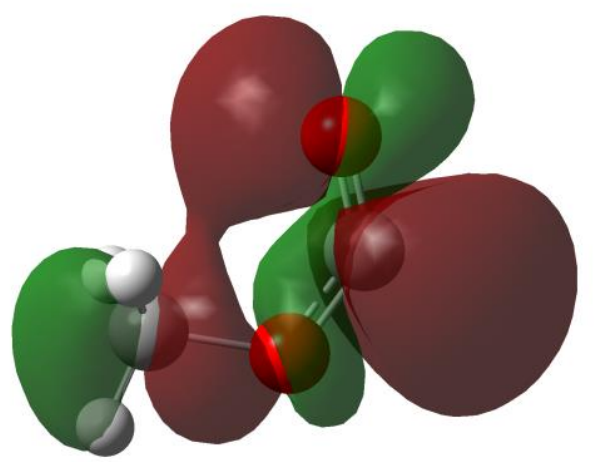

Fig. 4 Figure showing the SOMO of (a) acetaldehyde and (b) methyl formate radicals. There are two conformers for the methyl formate radical. The conformer shown is $4.55 \mathrm{kcal} \mathrm{mol}^{-1}$ lower in energy than the conformer where the methyl group is pointing down

There are various methods to calculate the ionisation energy of molecules. Here we have used Koopman's theorem applied to open shell system (i.e. IE = Eномо) considering that the Kohn-Sham (KS) potential is zero at infinity and therefore the ionisation energy of the KS system of a radical is by definition the negative of it SOMO energy, IE = - ESOMO. ${ }^{34}$ We have used the atomic polar tensor (APT) method to obtain atomic charges. The results we obtained for the IE and the ATP partial charge of acetaldehyde and methyl formate radical are shown in Table 1. 


\subsection{Calculation of IE and partial charges using DFT}

The relative permittivity, dipole moment, partial charge on the carbonyle carbon and ionisation energy of various solvents calculated using B3LYP/aug-ccpVTZ are tabulated in Table 1. We observe that there is a small effect of the dielectric constant on the IE and partial charge of the carbonyle carbon. The IE of the methyl formate radical increases from $152.28-153.43 \mathrm{kcal} \mathrm{mol}^{-1}$, which is higher than the IE of the acetaldehyde radical, which ranges between 128.60 to $129.29 \mathrm{kcal} \mathrm{mol}^{-1}$. The partial charge of the methyl formate radical increases from 0.838 to 0.975 , which is higher than the partial charge of the acetaldehyde radicals carbonyle carbon which ranges between 0.444 to 0.527 . The increased value of the IE and the partial charge of the methyl formate radical is a result of the larger positive inductive effect $(+I)$ that $\mathrm{CH}_{3}$ - has compared to $\mathrm{CH}_{3} \mathrm{O}$-, which has a negative (-I) inductive effect.

Table 1. Relative permittivity, dipole moment, partial charge on the carbonyle carbon and ionisation energy of various solvents calculated using UB3LYP/aug-cc-pVTZ.

\begin{tabular}{|c|c|c|c|c|c|c|}
\hline \multirow[b]{2}{*}{ Solvent } & \multirow{2}{*}{$\begin{array}{c}\text { Dielectric } \\
\text { constant }(\varepsilon)\end{array}$} & \multirow{2}{*}{$\begin{array}{c}\text { dipole } \\
\text { moment }(\boldsymbol{\mu}) \\
(\text { Debye }) \\
\end{array}$} & \multicolumn{2}{|c|}{ methyl formate radical } & \multicolumn{2}{|c|}{ acetaldehyde radical } \\
\hline & & & $\begin{array}{l}\boldsymbol{\delta}^{+} \\
(\mathrm{e}) \\
\end{array}$ & $\begin{array}{c}\text { IE } \\
\left(\mathrm{kcal} \mathrm{mol}^{-1}\right) \\
\end{array}$ & $\begin{array}{l}\boldsymbol{\delta}^{+} \\
(\mathrm{e}) \\
\end{array}$ & $\begin{array}{c}\mathbf{I E} \\
\left(\mathrm{kcal} \mathrm{mol}^{-1}\right) \\
\end{array}$ \\
\hline Cyclohexane & 2.0 & 0.00 & 0.838 & 152.38 & 0.444 & 128.60 \\
\hline Benzene & 2.3 & 0.00 & 0.850 & 152.47 & 0.451 & 128.65 \\
\hline chloroform & 4.8 & 1.15 & 0.910 & 152.93 & 0.487 & 128.93 \\
\hline THF & 7.4 & 1.73 & 0.935 & 153.12 & 0.503 & 129.07 \\
\hline Ethanol & 24.3 & 1.69 & 0.970 & 153.39 & 0.524 & 129.26 \\
\hline Methanol & 32.7 & 1.70 & 0.974 & 153.43 & 0.526 & 129.28 \\
\hline acetonitrile & 35.7 & 3.92 & 0.975 & 153.43 & 0.527 & 129.29 \\
\hline DMSO & 46.7 & 3.90 & 0.978 & 153.46 & 0.528 & 129.30 \\
\hline Water & 78.7 & 1.85 & 0.981 & 153.48 & 0.530 & 129.32 \\
\hline
\end{tabular}

The ionisation energy and the partial ATP charges are plotted in Fig. 5 and they show that the IE is linearly proportional to the ATP partial charge on the carbonyl carbon. This suggests that the larger the partial positive charge is on this carbon atom the smaller the tendency of the radical to form a carbocation as the ionisation energy will be larger. This may become quite useful in the study of reactions of radicals and their activity towards the formation of carbocations. 

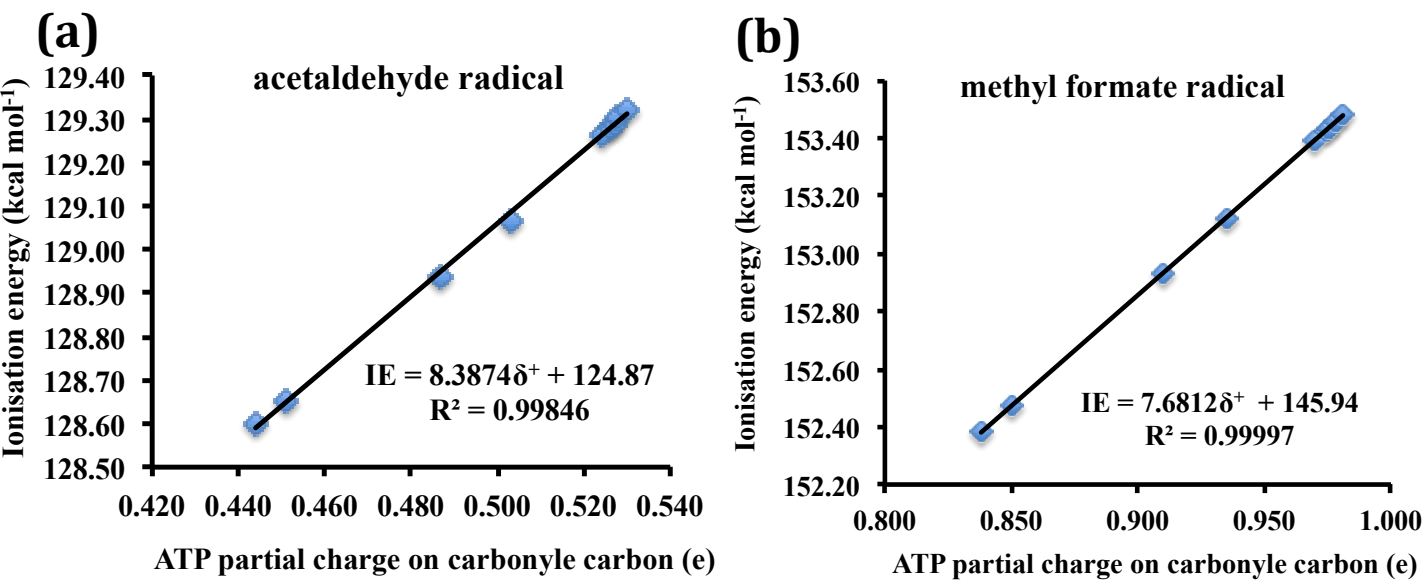

Fig. 5 Graph of the ionisation energy versus the partial charge on the carbonyle carbon for (a) acetaldehyde radical and (b) methyl formate radical using UB3LYP/aug-cc-pVTZ.

A perfect linear fit of the datapoints in Fig. 5 indicates that the ionisation energy of radicals can be predicted when the partial charges on the atoms are known. The origin of this linearity will be interpreted in section 3.4 using Coulomb's law a simple electrostatic model for the radical.

\subsection{Calculation of IE and partial charges using MP2}

The relative permittivity, dipole moment, partial charge on the carbonyle carbon and ionisation energy of various solvents calculated using UMP2/aug-ccpVTZ are tabulated in Table 2. We observe consistent to the earlier B3LYP results that there is a small effect of the dielectric constant on the IE and partial charge of the carbonyle carbon. The IE of the methyl formate radical increases from 261.05 $261.92 \mathrm{kcal} \mathrm{mol}^{-1}$, which is higher than the IE of the acetaldehyde radical, which ranges between $230.34-231.02 \mathrm{kcal} \mathrm{mol}^{-1}$. These IE are found to be significantly higher that those calculated using DFT. A similar large difference between the calculated IE using DFT and MP2 have been found in a previous study and it shows that MP2 is necessary to obtain accurate results as this can be seen in the calculated values of the methyl radical. ${ }^{35}$ These at B3LYP/6-311+G(2d,2p) are $149 \mathrm{kcal} \mathrm{mol}^{-1}$ compared to the MP2/6-311+G(2d,2p) value which is $241 \mathrm{kcal} \mathrm{mol}^{-1} .{ }^{35}$ The later compares very well to the adiabatic and vertical ionisation energy of the methyl radical, which was found to be $227 \mathrm{kcal} \mathrm{mol}^{-1}{ }^{36}$ Therefore we have repeated the DFT 
results using the UMP2, which includes electron correlation to larger degree than DFT.

The partial charge of the methyl formate radical increases from 0.827 to 0.980 which is higher than the partial charge of the acetaldehyde radicals carbonyle carbon which ranges between 0.359 to 0.439 . The trends observed in these results calculated using perturbation theory are identical with the trends observed calculated using hybrid density functional theory (DFT). This indicates that the inclusion of electron correlation does not significantly alter the correlation found and that higher correlated methods such as coupled cluster theory are not necessary to reach the conclusions reached in this study.

Table 2. Relative permittivity, dipole moment, partial charge on the carbonyle carbon and ionisation energy of various solvents calculated using UMP2/aug-cc-pVTZ.

\begin{tabular}{|c|c|c|c|c|c|c|}
\hline \multirow[b]{2}{*}{ Solvent } & \multirow[b]{2}{*}{$\begin{array}{c}\text { Relative } \\
\text { permittivity }\left(\varepsilon_{r}\right)\end{array}$} & \multirow[b]{2}{*}{$\begin{array}{c}\text { dipole } \\
\text { moment }(\boldsymbol{\mu}) \\
(\text { Debye }) \\
\end{array}$} & \multicolumn{2}{|c|}{ methyl formate radical } & \multicolumn{2}{|c|}{ acetaldehyde radical } \\
\hline & & & $\begin{array}{l}\boldsymbol{\delta}^{+} \\
(\mathrm{e})\end{array}$ & $\begin{array}{c}\text { IE } \\
\left(\mathrm{kcal} \mathrm{mol}{ }^{-1}\right)\end{array}$ & $\begin{array}{l}\boldsymbol{\delta}^{+} \\
\text {(e) }\end{array}$ & $\begin{array}{c}\text { IE } \\
\left(\mathrm{kcal} \mathrm{mol}^{-1}\right)\end{array}$ \\
\hline Cyclohexane & 2.0 & 0.00 & 0.827 & 261.05 & 0.359 & 230.34 \\
\hline Benzene & 2.3 & 0.00 & 0.840 & 261.13 & 0.365 & 230.40 \\
\hline chloroform & 4.8 & 1.15 & 0.903 & 261.50 & 0.398 & 230.68 \\
\hline THF & 7.4 & 1.73 & 0.930 & 261.65 & 0.412 & 230.80 \\
\hline Ethanol & 24.3 & 1.69 & 0.968 & 261.85 & 0.432 & 230.97 \\
\hline Methanol & 32.7 & 1.70 & 0.972 & 261.87 & 0.434 & 230.99 \\
\hline acetonitrile & 35.7 & 3.92 & 0.973 & 261.88 & 0.435 & 231.00 \\
\hline DMSO & 46.7 & 3.90 & 0.976 & 261.89 & 0.437 & 231.01 \\
\hline Water & 78.7 & 1.85 & 0.980 & 261.92 & 0.439 & 231.02 \\
\hline
\end{tabular}

With the results coming form perturbation theory we observe again in Fig. 6 that there is a linear correlation of the IE as a function of the partial positive charge on the carbonyle carbon of the two radicals under examination. These results are consistent with the result calculated using DFT and therefore confirm that there is agreement with methods that have the explicit inclusion of electron correlation. The origin of this linearity will be interpreted in section 3.4 using Coulomb's law a simple electrostatic model for the radical. 
(a)

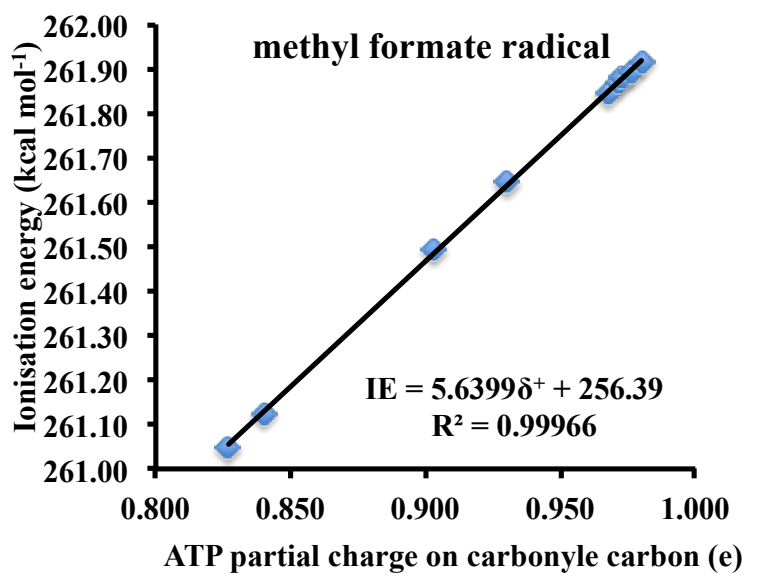

(b)

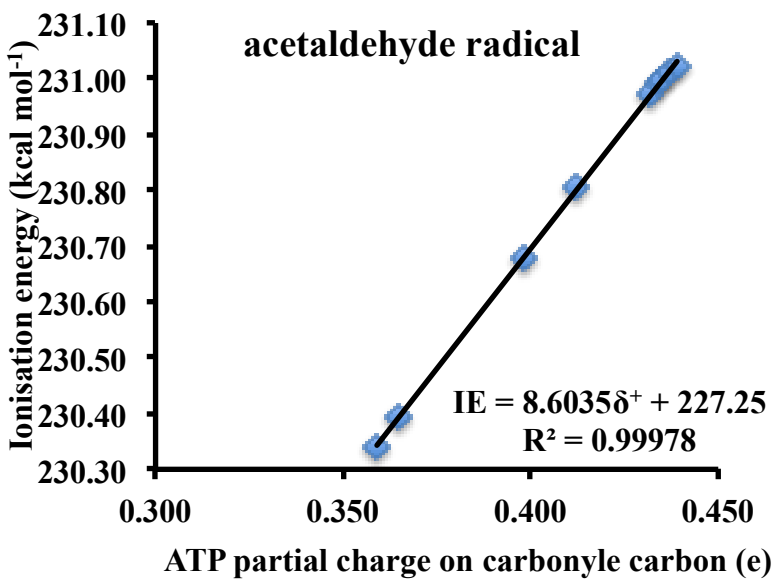

Fig. 6 Graph of the ionisation energy versus the partial charge on the carbonyl carbon for (a) methyl formate radical and (b) acetaldehyde radical using UMP2/aug-ccpVTZ.

\subsection{Simplified model that interprets the correlation between IE and partial charge}

In order to offer an interpretation of the linearity of the IE and the partial charges on the carbonyle carbon of acetaldehyde and methyl formate radical we consider an electrostatic model where atoms and the valence single electron residing in the singly-occupied-molecular-orbital of the two molecules are represented by point charges.

If the molecular system of a radical could be represented by point charges as shown in scheme 1.

\section{Radical}

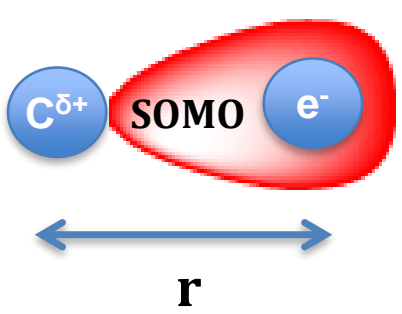

\section{Carbocation}

\section{IE}

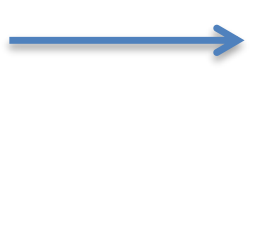

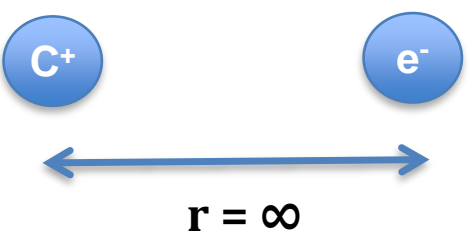

Scheme 1. Showing the elementary charges and their inter-particle separation during the formation of a carbocation from a radical

Then atoms that have positive partial charges (i.e. $\delta^{+}$) will require more energy to give away one of their valence electrons as there is an attractive energy between the 
effective nuclear charge of the atom and the electrons in the valence shell of the molecule given by Coulomb's law,

$$
U=\frac{q^{\delta+} q^{e} e^{2}}{4 \pi \varepsilon_{0} \varepsilon_{r} r} \quad()
$$

, where $r$ is the separation between the point charge $\left(q^{\delta+}\right)$ of the atom and the charge of the electron $\left(q^{e}\right), e$ is the elementary charge of an electron $\left(1.602 \times 10^{-19} \mathrm{C}\right), \varepsilon_{0}$ is the absolute permittivity of vacuum $\left(8.854 \times 10^{-12} \mathrm{Fm}^{-1}\right)$ and $\varepsilon_{r}$ the dielectric constant of the solvent (e.g. $\left.\varepsilon_{r}\left(\mathrm{H}_{2} \mathrm{O}\right)=78.7\right), q^{\delta+}$ the partial positive charge of the atom on which the lone electron resides and $q^{e}=-1$. We can also estimate the force between the effective nuclear charge of the atom and the electron by taking the negative derivative of the Coulomb potential with respect to $r$ given by the following equation,

$$
F=-\frac{\partial U}{\partial r}=\frac{q^{\delta+} q^{e} e^{2}}{4 \pi \varepsilon_{0} \varepsilon_{r} r^{2}} \quad(N)
$$

Since the ionisation energy is essentially the Coulomb potential at infinite separation minus the Coulomb potential at an inter-particle separation of $\mathrm{r}$ according to Scheme 1,

$$
I E=U_{\infty}-U_{r}
$$

and $U_{\infty}=0$ and $q^{e}=-1$, therefore,

$$
I E=\frac{e^{2}}{4 \pi \varepsilon_{0} \varepsilon_{r} r} \cdot q^{\delta+}(J)
$$

For a molecular system of a radical as the shape and dimensions of the SOMO are roughly the same, for the two radicals, as can be seen in Fig. 3, therefore for the same solvent all parameter in Eqn. 4 become a constant apart from the partial charge of the carbon atom. Therefore,

$$
I E=\text { constant } \cdot \frac{q^{\delta+}}{\varepsilon_{r}}(J)
$$

which explains the linear correlation found in the plots of the ionisation energy versus the partial positive charge of the $\alpha$ carbon of radicals devided by the dielectric constant of the solvent which are given in Fig. 7. 


\section{(a)}

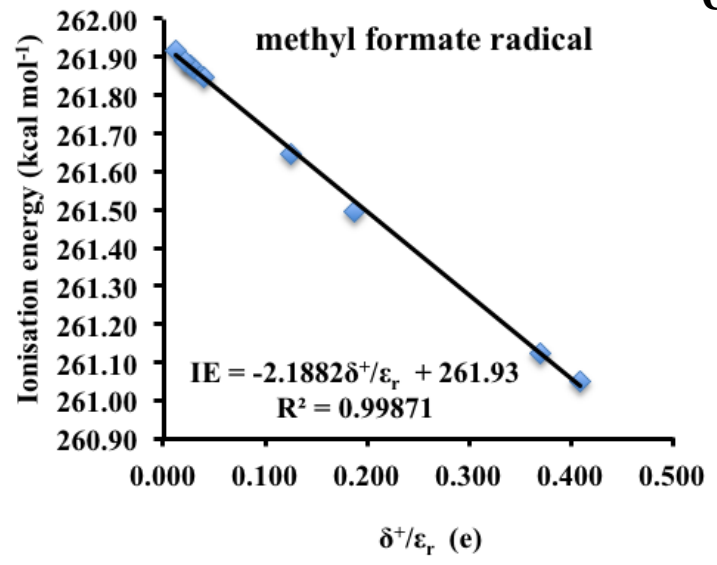

(b)

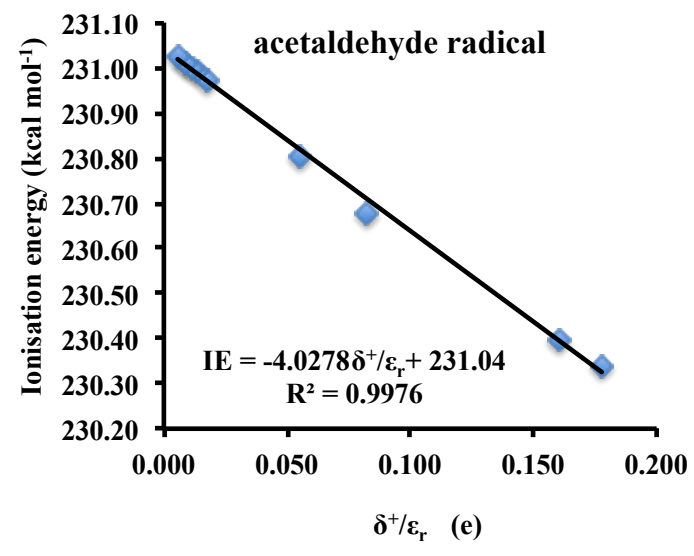

Fig. 7 Graph of the ionisation energy versus the partial charge on the carbonyle carbon devided by the dielectric constant of the solvent for (a) the methyl formate radical and (b) the acetaldehyde radical calculated using UMP2/aug-cc-pVTZ.

\subsection{Solvent effect on partial charge of carbonyle carbon}

A Solvent effect on the rate of radical to carbocation formation is not known currently in the literature. However nucleophilic substitution reactions $\left(\mathrm{S}_{\mathrm{N}} 1\right.$ and $\left.\mathrm{S}_{\mathrm{N}} 2\right)$ are strongly affected by solvent effects. Polar aprotic solvents (e.g.DMF, DMSO, HMPA, Acetonitrile) are known to enhance the rate of $\mathrm{S}_{\mathrm{N}} 2$ reactions because they dissolve many salts solvating only the metal ion whereas the anion remains free to perform nucleophilic substitutions. Whereas, polar -OH and - $\mathrm{NH}$ containing solvents are generally not as effective as they coordinate with their $\mathrm{H}^{\delta^{+}}-\mathrm{X}$ moeties to the nucleophile, $\mathrm{X}^{-}$, reducing the negative charge on it, decreasing therefore its nucleophilicity.

In $S_{N} 1$ mechanism polar solvent such as water and methanol stabilise the formation of the carbocation by forming a solvation sphere where the negative point of the solvent dipole moment is pointing towards the carbocation. This increases the lifetime of the carbocation and therefore increases the rate of nucleophilic substitution.

In this study we find that there is a significant effect of the dielectric constant of the solvent on the partial positive charge of the carbonyle carbon. In particular using UMP2/aug-cc-pVTZ(5d, 7f) the $\delta+$ increases from 0.359 to 0.439 for the acetaldehyde radical and for the methyl formate radical from 0.827 to 0.980 as the 
relative perimittivity of the solvents changes from 2.0 (cyclohexane) to $78.7\left(\mathrm{H}_{2} \mathrm{O}\right)$. This clearly suggests that the dielectric constant enhances the positive charge of the carbonyle carbon, which then increases the ionisation energy of the radicals and therefore decreases the rate of the carbocation formation. It is therefore suggested that solvents with high dielectric constant would have a solvent effect on the carbocation formation shown in scheme 1 and should therefore be the prefered option to enhance the rate of carbocation formation. It is noted though that this solvent effect is consistent for both the methyl formate and the acetaldehyde radical, it is small, as a change of the IE going from the low dielectric constant to the high dielectric constant the solvent affect it by less than $1 \mathrm{kcal} \mathrm{mol}^{-1}$. It is noted that electron solvation has not been considered in this model, which may lead to different trends.

\section{Conclusions}

Through DFT and MP2 calculations we find that the methyl formate radical forms carbocations to a smaller degree than the acetaldehyde radicals. This is because its ionisation energy ranges between 261.05 to $261.92 \mathrm{kcal} \mathrm{mol}^{-1}$ whereas that of the acetaldehyde radical ranges between 230.34 to $231.02 \mathrm{kcal} \mathrm{mol}^{-1}$. We find a linear correlation between the IE and the partial charge on the carbonyle carbon atom, which has the singly occupied molecular orbital. Such a relationship between IE and partial charge is useful to rationalise the rate of carbocation formation from radicals and for reactions of these radicals with other molecules. A solvent effect is observed which enhances the partial charge and the IE based on a simplified point charge model following Coulomb's law.

\section{Conflicts of Interest}

The authors declare no conflicts of interest

\section{Acknowledgements}

Computation for the work presented in this paper was supported by the University of Greenwich High Performance Computer resources (https://www.gre.ac.uk/it-and-library/hpc). 


\section{References}

1. N. A. Weires, Y. Slutskyy and L. E. Overman, Angew. Chemie Int. Ed., 2019, 58, 8561-8565.

2. Y. Nunes, G. Martins, N. J. Mason, D. Duflot, S. V. Hoffmann, J. Delwiche, M. J. Hubin-Franskin and P. Limão-Vieira, Phys. Chem. Chem. Phys., 2010, 12, 15734-15743.

3. E. Jiménez, M. Antiñolo, B. Ballesteros, A. Canosa and J. Albaladejo, Phys. Chem. Chem. Phys., 2016, 18, 2183-2191.

4. W. Reutemann and H. Kieczka, Ullmann's Encyclopedia of Industrial Chemistry, 2000, DOI: doi:10.1002/14356007.a12_013

10.1002/14356007.a12_013.

5. W. Couteau and J. Ramioulle, US Patent US4216339.

6. D. A. Good and J. S. Francisco, J. Phys. Chem. A, 2002, 106, 1733-1738.

7. T. J. Wallington, M. D. Hurley, T. Maurer, I. Barnes, K. H. Becker, G. S. Tyndall, J. J. Orlando, A. S. Pimentel and M. Bilde, J. Phys. Chem. A, 2001, 105, 5146-5154.

8. J. C. Hansen, Y. Li, J. S. Francisco, J. J. Szente and M. M. Maricq, J. Chem. Phys., 2000, 113, 6465-6468.

9. N. L. Arthur and P. Gray, Trans. Farad. Soc., 1969, 65, 424-433.

10. T. R. Donovan, W. Dorko and A. G. Harrison, Can. J. Chem., 1971, 49, 828832.

11. M. J. Abplanalp and R. I. Kaiser, Phys. Chem. Chem. Phys., 2019, 21, 1694916980.

12. P. C. Arroyo, K. T. Malecha, M. Ammann and S. A. Nizkorodov, Phys. Chem. Chem. Phys., 2018, 20, 30021-30031.

13. A. W. Harrison, A. Kharazmi, M. F. Shaw, M. S. Quinn, K. L. K. Lee, K. Nauta, K. N. Rowell, M. J. T. Jordan and S. H. Kable, Phys. Chem. Chem. Phys., 2019, 21, 14284-14295.

14. G. M. Thiele, M. J. Duryee, M. S. Willis, D. J. Tuma, S. J. Radio, C. D. Hunter, C. S. Schaffert and L. W. Klassen, Alcoholism: Clinical and Experimental Research, 2010, 34, 2126-2136.

15. C. S. Lieber, Pharmacology \& Therapeutics, 1990, 46, 1-41.

16. R. Nicholls, J. de Jersey, S. Worrall and P. Wilce, International Journal of Biochemistry, 1992, 24, 1899-1906.

17. F. Bootorabi, J. Jänis, V. P. Hytönen, J. Valjakka, M. Kuuslahti, D. Vullo, 0. Niemelä, C. T. Supuran and S. Parkkila, Journal of Enzyme Inhibition and Medicinal Chemistry, 2011, 26, 862-870.

18. C. D' Addario, Y. Ming, S.-0. Ögren and L. Terenius, The FASEB Journal, 2007, 22, 662-670.

19. J. E. Klaunig, Z. Wang, X. Pu and S. Zhou, Toxicology and Applied Pharmacology, 2011, 254, 86-99.

20. E. Albanoa, P. Clot, A. Comoglio, M. U. Dianzani and A. Tomasi, FEBS Letters, 1994, 348, 65-69.

21. M. Head-Gordon, J. A. Pople and M. J. Frisch, Chem. Phys. Lett., 1988, 153, 503-506.

22. M. J. Frisch, M. Head-Gordon and J. A. Pople, Chem. Phys. Lett., 1990, 166, 275-280.

23. M. J. Frisch, M. Head-Gordon and J. A. Pople, Chem. Phys. Lett., 1990, 166, 281-289. 
24. A. D. Becke, J. Chem. Phys., 1993, 98, 5648.

25. C. Lee, W. Yang and R. G. Parr, Phys. Rev. B, 1988, 37, 785-789.

26. D. E. Woon and T. H. Dunning Jr., J. Chem. Phys., 1993, 98, 1358.

27. A. Wilson, T. van Mourik and T. H. Dunning Jr., J. Mol. Struct., 1996, 388, 339-349.

28. K. A. Peterson, D. E. Woon and T. H. Dunning Jr., J. Chem. Phys., 1994, 100, 7410.

29. R. A. Kendall and T. H. Dunning Jr., J. Chem. Phys., 1992, 96, 6796.

30. T. H. Dunning Jr., J. Chem. Phys., 1989, 90, 1007.

31. S. Miertuš, E. Scrocco and J. Tomasi, Chem. Phys., 1981, 55, 117-129.

32. J. L. Pascual-ahuir, E. Silla and I. Tuñon, J. Comp. Chem., 1994, 15, $1127-$ 1138.

33. J. Cioslowski, J. Am. Chem. Soc., 1989, 111, 8333-8336.

34. A. Savin, C. J. Umrigar and X. Gonze, Chem. Phys. Lett., 1998, 288, 391-395.

35. B. S. Jursic, Journal of Molecular Structure: THEOCHEM, 1998, 432, 211217.

36. F. A. Houle and J. L. Beauchamp, J. Am. Chem. Soc., 1979, 101, 4067-4074. 